\title{
Evaluación clínica para el parto después de cesárea
}

\author{
Doctores Fernando Del Corral y Saulo Muñoz Delgado \\ Clínica de Maternidad de Cali. Colombia.
}

Las nuevas indicaciones de la cesárea, el mejoramiento de sus técnicas y por esto su utilización corriente, ha producido un número cada vez mayor de pacientes sometidas a esta intervención, y como consecuencia un aumento del problema del embarazo y el parto después de cirugía.

Este aspecto obstétrico, de por sí serio, se ve agravado en nuestro medio por el impeđimento religioso, ético y social para hacer la ligadura de las trompas o para usar cualquier medio anticonceptivo o contraceptivo en los casos especiales que nos ocupan.

Las estadísticas de los partos atendidos en la Clínica de Maternidad de Cali durante los años de 1956, 1957 y hasta agosto de 1958, demuestran el aumento progresivo de pacientes con cesárea anterior, hasta el punto de que en lo que llevamos corrido del año se ha superado a los anteriores. Asímismo se nota el aumento de cesáreas durante estos años.

CESAREA Y EMBARAZO DESPUES DE CESAREA

\begin{tabular}{cccc}
\hline Año & No de partos & Cesáreas & Cesáreas ant. \\
\hline 1956 & 3149 & 128 & 34 \\
1957 & 2403 & 172 & 43 \\
1958 (agosto) & 2320 & 108 & 48 \\
\hline
\end{tabular}


Los peligros que constituyen para la paciente esta intervención en el siguiente embarazo, asi como la divergencia sobre el manejo de estos casos, nos obliga a hacer una revisión cuidadosa ciel tema.

Debemos considerar primeramente las complicaciones a que se ven sometidas dichas pacientes y luego haremos una evaluación clínica acerca de la conducta que se debe seguir en estos casos.

\section{I-COMPLICACIONES}

A) Mortalidad materna y fetal.-Intentar un parto después Cie una cesárea es un procedimiento peligroso, aun con la paciente más cuidadosamente seleccionada, y la hospitalización más conveniente. Sin embargo, este riesgo no es mayor para madre y niño que aquel que se presenta con la cesárea de repetición. Por ctro lado el riesgo parece ser disminuído para la futura madre cespués de un parto subsiguiente a la cesárea, más que en los casos en que se hace cesárea electiva de repetición. Cosgrove (12-13), en su estadística presenta 175 casos de mujeres con cesáreas previas que tuvieron 221 niños por parto sin muerte, debida a ruptura uterina. McLean (33) presenta las mismas conclusiones en 43 casos.

Duckering (16), en 1946, informa su experiencia durante 11 años, en los cuales obtuvo el $42 \%$ de partos subsiguientes a la cesárea sin mortalidad materna o fetal. En otro artículo Duckering presenta 78 pacientes que tuvieron partos sobre 253 cesáreas anteriores con una mortalidad fetal de 5.8 y una mortalidad materna de $0.67 \%$.

Fleming (19) presenta 150 casos de parto con solo una muerte materna después del trabajo de parto (hemorragia postparto), 1 muerte fetal debida a ruptura cicatricial que pudo haber sido evitada por una cesárea electiva de repetición.

Es bastante difícil evaluar la estadística global debida a los métodos diferentes para obtenerla. La mortalidad fetal después de ruptura promedia de 0 a $8 \%$ según diferentes autores que han escrito acerca de este tema. La mortalidad materna promedia cie 0 a $0.67 \%$ y es mucho más alta en los casos de cesárea de repetición. Nosotros debemos concluir que después de una cesárea los embarazos subsiguientes aumentan las posibilidades de complicaciones que no pueden ser evitadas por la cesárea de repetición o el parto; que en la mayoría de los casos de rúptura del átero la responsabilidad de la muerte fetal debe acharcarse al 
médico general que permite evolucionar casos complicados y los conduce a puntos peligrosos.

En las estadísticas de la Clínica de Maternidad durante los años 1956, 1957 y parte de 1958, el único caso fatal después de cesárea fue debido a abuso de ocitócicos en una paciente atendida en el domicilio, que ingresó a la clínica con un cuadro de ruptura uterina, en anemia aguda, shock y que falleció a los $20 \mathrm{mi}$ nutos.

Entre los casos seleccionados para cesárea de repetición o parto no se presentó mortalidad materna. La mortalidad fetal se limitó a 3 niños, 2 de los cuales en casos de rúptura completa y uno entre las pacientes con cesárea de repetición.

MORTALIDAD MATERNA Y FETAL

\begin{tabular}{|c|c|c|c|}
\hline & & Materna & Fetal \\
\hline $\begin{array}{l}\text { Cesárea de repetición .... . . } \\
\text { Parto después de cesárea: }\end{array}$ & $\cdots \cdots$ & 0 & 1 \\
\hline $\begin{array}{l}\text { Pacientes seleccionadas } \\
\text { Atendido en el domicilio }\end{array}$ & $\begin{array}{c}\cdots \\
\text { (ingresó con rup- }\end{array}$ & 0 & 2 \\
\hline tura uterina) $\ldots \ldots$ & $\ldots \ldots \ldots$ & 1 & 1 \\
\hline
\end{tabular}

CUADRO NUMERO 2

El médico general ignora el hecho de que la profilaxis en estos casos comienza mucho más temprano y que no debe permitirse trabajo de parto en casos sin selección y sin un criterio exacto para poder reconocer estas complicaciones. En nuestros casos aún es mucho más dramática esta situación, ya que un gran porcentaje de pacientes llegan a la clínica con un trabajo cie parto avanzado o después de haber sido sometidas a ocitócicos o maniobras y la mayoría de ellas sin ningún control prenatal.

CONTROL PRENATAL

\begin{tabular}{rrrrrrrrrrrrr}
1956 & $\ldots$ & $\ldots$ & $\ldots$ & $\ldots$ & $\ldots$ & $\ldots$ & $\ldots$ & $\ldots$ & $\ldots$ & $\ldots$ & 4 \\
1957 & $\ldots$ & $\ldots$ & $\ldots$ & $\ldots$ & $\ldots$ & $\ldots$ & $\ldots$ & $\ldots$ & $\ldots$ & $\ldots$ & 8 \\
1958 & (hasta agosto) & $\ldots$ & $\ldots$ & $\ldots$ & $\ldots$ & $\ldots$ & 13 \\
\hline
\end{tabular}


B) Ruptura uterina.-La mayor complicación es la ruptura del útero durante el trabajo de parto. Para un mejor entendimiento debemos tener en cuenta ciertos hechos anatómicos del útero durante el embarazo en los casos de una cesárea previa. La porción segmentaria uterina se aumenta verticalmente y al mismo tiempo se adelgaza a medida que el trabajo de parto progresa, de tal manera que la "retracción del anillo de Bandl" es un hecho precursor de la ruptura durante el trabajo de parto en los casos de cesárea anterior. Es lógico creer que una cicatriz corporal está expuesta a los efectos de cicatrización de un útero sometido a períodos de contracción activa y de descanso durante su involución. Esta cicatriz sobre la zona motora del útero sufre cambios vasculares substanciales que la hacen defectuosa.

La cesárea clásica con su peritonización imperfecta permanece en contacto con muchas de las estructuras abdominales y esto puede resultar en la formación de adherencias, las cuales van a producir una resistencia a la contracción uterina, aumentando las posibilidades de desgarro en el tejido cicatricial (36). La mayoría de los autores están de acuerdo en la alta incidencia de rupturas uterinas en los subsiguientes embarazos debido a este tipo particular de cesárea. Wilson (50) encuentra que sobre la incisión corporal 56\% de ellas presentaron defectos en la cicatriz, deduciendo que la cicatriz segmentaria baja en sentido transversal "es alrededor de 4 veces más segura para posteriores embarazos". Fleming (19) anota que en tales casos la inserción placentaria baja se hace frecuentemente sobre el área cicatricial y por lo tanto es más posible la ruptura. Hindman (24) en su estadística muestra que la ruptura de la cicatriz de la cesárea clásica ocurre 16 veces más frecuentemente que en los casos de cesárea segmentaria baja. Con el fin de hacer un cuadro comparativo de la incidencia de rupturas en las mujeres con cesárea en el subsiguiente embarazo y que llegan al parto, presentamos las siguientes estadísticas: 


\begin{tabular}{|c|c|c|c|}
\hline Autores & $\begin{array}{l}\text { Estadísticas } \\
\text { Casos }\end{array}$ & $\begin{array}{l}\text { Rupturas del Porc } \\
\text { útero }\end{array}$ & orcentajes \\
\hline McLean (12) $\quad \ldots \quad \ldots, \quad \ldots, \ldots c c c$ & 43 & 0 & $0.0 \%$ \\
\hline 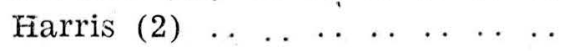 & 18 & 0 & $0.0 \%$ \\
\hline 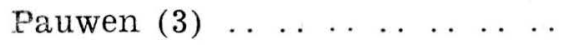 & 148 & 1 & $0.6 \%$ \\
\hline Winchester and Browne (4) .. & 136 & 1 & $0.7 \%$ \\
\hline Duckering (5) $\quad \ldots \begin{array}{llllll} & \ldots & \ldots & \ldots & \ldots & \ldots\end{array}$ & 445 & 6 & $1.3 \%$ \\
\hline 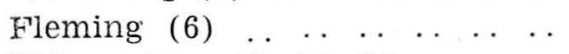 & 150 & 2 & $1.3 \%$ \\
\hline Wilson (New York) (7) $\ldots \ldots \ldots$ & 498 & 8 & $1.0 \%$ \\
\hline Schmitz and Baba (8) & 51 & 3 & $1.9 \%$ \\
\hline Schmitz (9) & 190 & 6 & $3.1 \%$ \\
\hline Schmitz and Gaywski (10) . . . & 62 & 6 & $3.2 \%$ \\
\hline Holland (11) & 448 & 18 & $4.0 \%$ \\
\hline Bruce (Glasgow, Scotland) (12) & 47 & 2 (clásica) & $4.2 \%$ \\
\hline De Anda (México) (13) $\ldots \ldots \ldots$ & 35 & 3 & $6.7 \%$ \\
\hline Hindman (Boston) (14) . . & 118 & 8 & $6.7 \%$ \\
\hline
\end{tabular}

1. Am. J. Obst. \& Gynec. 60:860, 1950.

2. Am. J. Obst. \& Gynec. Vol. 71:6:1.202, 1956.

3. Zentralbl F. Bynak. 69:110, 1957.

4. Exerte Med. (Soc. X) 1954, 7:248.

5. Am. J. Obst. Gynec. 51:621, 1946.

6. Am. J. Obst. Gynec. 1955; 70:923.

7. Am. J. Obst. Gynec. 62:1.225, 1951.

8. Am. J. Obst. Gynec. 57:669, 1949.

9. Supp. Vol. Am. J. Obst. \& Gynec. Gia; 289: 1951.

10. (J. M. M. M.). Chicago, 1951.

11. Obst. Gynec. 1921; 28:358.

12. Am. J. Obs. \& Gynec. Vol. 66. No 6:1.191, 1953.

13. Ginec. Obst. México, 1953; 8:336.

14. Am. J. Obst. \& Gynec. 55:273; 1948.

De estas estadísticas podemos deducir que el promedio de zupturas uterinas en pacientes sometidas a parto vaginal es de $2.4 \%$. Aquí debemos reconocer que hay dos clases diferentes de rupturas uterinas (12-13).

Primeramente la ruptura catastrófica con la salida del feto dentro de la cavidad peritoneal. Segundo: una pobre cicatrización de la herida original en la cual el contenido del útero no se ha salido y los efectos maternos son casi nulos. 
El primer cuadro se ve frecuentemente en los casos de rupturas de cicatrices de cesáreas clásicas, mientras el segundo generalmente es asintomático y el feto casi nunca se compromete. Este tipo generalmente se diagnostica durante la cesárea de repétición o a la exploración uterina después del parto vaginal.

Es importante saber que en casi todos los casos de esta última clase no es necesario tratamiento alguno después del parto. Éstos tipos de rupturas se consideran como incompletas cuando solamente los filos del miometrio están cubiertos por una delgada membrana relativamente avascular de tejido cicatricial (38).

El siguiente cuadro esquemático muestra los diferentes tipos de las rupturas arriba mencionadas durante el embarazo:

2

Dehiscencia incompleta.
Endometrio

Miometrio

Peritoneo

En muchos casos de las rupturas incompletas o silenciosas solamente las capas vesicales y la reflexión peritoneal mantienen el producto de la concepción dentro de la cavidad uterina.

Presentamos en seguida el cuadro de Pedowitz y Schwartz (38) para mostrar la incidencia de ruptura uterina en relación al tipo de cesárea:

RUPTURAS

\begin{tabular}{|c|c|c|c|c|c|c|c|}
\hline Tipo de incísión uterina & & & Total & Completa & $\begin{array}{l}\text { Incom- } \\
\text { pleta }\end{array}$ & $\begin{array}{l}\text { Total de } \\
\text { rupturas }\end{array}$ & $\begin{array}{c}\text { Incldencia } \\
\text { de ruptu- } \\
\text { ras } \% / 0\end{array}$ \\
\hline Segmentaria transversa & . & . . & . 266 & 19 & 3 & 22 & 8.3 \\
\hline Segmentaria longitudinal & . & . . & . 155 & 16 & 4 & 20 & 12.9 \\
\hline 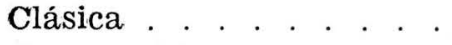 & . & . . & 33 & 5 & 1 & 6 & 18.2 \\
\hline Desconocida . . . . . . . & . & . . & 28 & 0 & 0 & 0 & 0.0 \\
\hline Totales & . . & . . & . 482 & 40 & 8 & 8 & $10.0 \%$ \\
\hline
\end{tabular}

CUADRO NUMERO 4

P. Pedowitz, and Schwartz. Am. J. of Obst. \& Gynec., Volumen 74, 5: $1.071,1957$. 
Como podemos ver, los casos dramáticos casi siempre se presentan en la cesárea clásica más que en la ruptura de cesárea segmentaria baja.

Las estadísticas de la Clínica de Maternidad nos muestran 2 rupturas completas y 4 incompletas en 63 casos que fueron atendidos por parto después de cesárea. Creemos conveniente analizar detenidamente los casos de rupturas completas.

Historia clinica número 13-5-57.

Edad: dieciocho años.

Ingresó: octubre 4 de 1957 a las 22 horas.

Grávida: 2 para 0 con antecedente de cesárea intervenida por médico general el año de 1956.

Institución: fuéra de la Clínica de Maternidad.

Indicación: desconocida.

Examen obstétrico.-Octubre 4/57. Horas: 22. Altura uterina, 31 centímetros. Latidos fetales positivos. Contracciones irregulares de poca intensidad y duración. Tacto vaginal: dilatación, 1 centímetro. Borramiento cuello, 30\%. Membranas rotas espontáneamente, prematuramente hace 6 horas. Presentación OIA. Estación de la cabeza: - 1 .

A las 0.30 horas del 5 de octubre inicia contracciones de buena intensidad y duración y a la 1.55 presenta ruidos arrítmicos con hipertonía uterina y dolor en la región hipogástrica. Se traslada a sala de cirugía y se interviene con diagnóstico de ruptura uterina y muerte fetal. Al abrir la cavidad se encuentra el feto y la placenta en el abdomen y ruptura del útero en forma de $\mathbf{T}$ en la porción segmentaria. Se practica cirugía reparadora. Feto de 3.550 gramos, muerto.

\section{Historia clínica número 327-4-58.}

Edad: treinta y cinco años.

Ingresó: abril 17 de 1958 a la 1.45 horas.

Causa de ingreso: grávida 4 para 2 con embarazo a término en trabajo de parto y antecedente de cesárea en el último embarazo por placenta previa practicada en la Clínica de Maternidad en diciembre de 1956.

Examen obstétrico.-Abril 17/58, hora: 1.45. Altura uterina, 33 centímetros. Latidos fetales, 150 . Contracciones uterina espaciadas de buena intensidad y duración. Tacto vaginal: dilatación, 2 centimetros. Borramiento, 60\%. Membranas integras. Pre- 
sentación cefálica. Estación de la cabeza: - 2. Edad del embarazo, más o menos, 40 semanas.

Conducta.-Prueba de trabajo de más o menos 6 horas.

Evolución.-A las 5 a. m. paciente sudorosa, pálida, con dolor en el hipogastrio, pulso de 120 y hemorragia vaginal de más o menos 200 c. c. Distensión abdominal. Se traslada a cirugía, encontrándose una ruptura uterina corporo-segmentaria de más o menos 12 centímetros. Se practica cirugía reparadora. Feto muerto y placenta en cavidad abdominal. Feto de 3.660 gramos.

En el primer caso se trataba de una paciente nulípara, mal seleccionada, debido a que se desconocía la institución y el cirujano que había practicado la intervención, así como el curso postoperatorio. La pelvis era amplia y el no haber iniciado trabajo de parto franco, posiblemente fue la causa para permitirle unas horas de trabajo de prueba. La ruptura se produjo casi a los $60 \mathrm{mi}-$ nutos de haberse iniciado el trabajo de parto, cuando aún la dilatación del cuello no había alcanzado más de 2 centímetros. Otra de las causas de error fue el cuello inmaduro con que se permitió el trabajo de prueba. Este caso, con el criterio establecido hoy en día, no lo hubiéramos seleccionado para un parto por las causas anteriormente anotadas.

El caso número 2, multípara con su última cesárea por placenta previa en la Clínica de Maternidad, era un caso que reunía todas las condiciones para permitirle un trabajo de parto. Sus antecedentes quirúrgicos eran aceptables y el examen obstétrico, como la valoración de la cicatriz, permitía un trabajo de prueba. Es posiblemente el único caso que pudiéramos considerar al sacar los índices de mortalidad depurada, como responsable de ruptura durante el trabajo de prueba.

Las rupturas incompletas (dehiscencias) las analizamos detenidamente en el capítulo de la evaluación de la cicatriz después del parto. Su tratamiento es conservador y en nuestros casos no se presentó complicación alguna.

\section{II.-CONDUCTA DESPUES DE LA CESAREA}

Sobre la forma como debe conducirse la paciente embarazada después de una cesárea existen aún divergencias, por lo cual consideramos de interés resumir las opiniones de algunos importantes Centros, con el fin de ilustrar las conductas establecidas hasta el presente. 
Bak y Hayden (2) 1955, después de estudiar 52 casos de ruptuura uterina en 71.473 partos en Chicago Lying in Hospital establecen en una forma segura que ellos creen "que después de una cesárea debe verificarse siempre cesárea de repetición".

Wilson (50) 1951, después de revisar este punto en el Deparamento de Obstetricia y Ginecología de Cornell University $\mathrm{Me}$ dical College y el Women's Hospital de Nueva York, dan importancia a la conducta individual con la evaluación cuidadosa de cada uno de los casos. Encontraron que en las pacientes que fueron programadas para cesárea de repetición se presentaron unas pocas rupturas antes de la intervención.

Donald y Frazer (18) 1953, expresaron adecuadamente su punto de vista según el cual la cesárea de repetición está muy Iejos de ser la regla aceptada o practicada en Inglaterra.

Birbaum (6) 1956, establece en el New York Lying in Hospital la cesárea de repetición como la regla en la práctica privada. y en cambio permite el parto en los servicios hospitalarios.

En los dos hospitales de enseñanza obstétrica de Adelaide (39), el cie Maternidad Queen Victoria y el Queen Elizabeth, no ha habido casos de ruptura del útero después de cesárea en los últimos cinco años. Durante este período, entre 17.000 partos, 216 mujeres presentaron una historia de cesáreas previas antes del parto. De éstas, 83 se condujeron en forma segura con parto; a las otras 133 se les practicó cesárea de repetición, teniendo una gran proporción de ellas nuevas indicaciones de cesárea. Creen que la conducta debe ser la selección individual después de diversas consideraciones, tales como la cesárea previa, la edad de la paciente, el tipo de pelvis, la clase de cesárea, la convalescencia, las contracciones y otros datos de gran importancia en la historia clinica.

El parto vaginal después de cesárea previa, de acuerdo con Schmitz $(42,43,44)$, Cosgrove $(12,13)$, Avilés (1), es en muchos casos un procedimiento racional y debe ser cuidadosamente considerado antes de practicar nueva cirugía.

Gran número de autores $(8,13,24,25,43,44)$ creen que el cbstetra que intenta un trabajo de parto en pacientes con cesária previa, cuyas condiciones lo permiten, corre menos riesgo materno que en los casos en que se practica cesárea de repetición. Presentan estadísticas de varios centros hospitalarios que nos comprueban esta conducta. 
EXPERIENCIA HOSPITALARIA CON PARTO DESPUES DE CESAREA

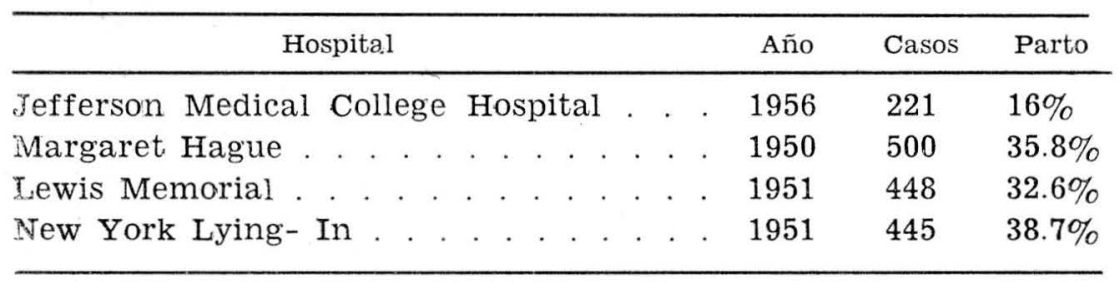

CUADRO NUMERO 5

De acuerdo con estas estadísticas creemos que una cuidadosa evaluación de cada caso, más que una rutina en la repetición de la cesárea, dará a muchas mujeres una oportunidad para demostrar su capacidad para el parto. Otra razón poderosa es la de que la cesárea produce tres veces mayor mortalidad que el parto (29), así como mayor riesgo de ruptura para el próximo embarazo. Los progresos obstétricos en los últimos cinco años, tales como el avance con los rayos $\mathrm{X}$, placentografía, pelvimetría, histerografía $\mathrm{y}$, además, el desarrollo de técnicas operatorias empleando la porción segmentaria uterina, así como una mejor información médica, han hecho posible para mayor número de mujeres esperar un parto después de cesárea.

De las consideraciones anteriores tenemos que existen dos formas de tratar a la paciente embarazada con cesárea anterior:

a) Cesárea de repetición.

b) Parto después de cesárea.

En la Clínica de Maternidad de Cali, en los años de 1956 y 1957 hasta agosto de 1958, hemos considerado cada caso indiviriualmente, por lo cual presentamos las estadísticas y las normas que hemos seguido:

TIPO DE PARTO EN PACIENTES CON CESAREA PREVIA (Maternidad de Cali)

\begin{tabular}{|c|c|c|c|}
\hline & 1956 & 1957 & 1958 ( 8 meses) \\
\hline Total de pacientes . . . . . & 3.149 & 3.403 & 2.320 \\
\hline Cesárea de repetición . . . . . & 20 & 18 & 24 \\
\hline Parto después de cesárea . . . . & 14 & 25 & 24 \\
\hline
\end{tabular}




\section{A) Cesárea electiva de repetición}

Hay muchas pacientes para quienes un trabajo de prueba está definitivamente contraindicado; tales son los casos de "indicaciones absolutas" para la cesárea anterior. Estos casos deben ser cuidadosamente vigilados durante el embarazo e instruídos apropiadamente para cualquier posible anormalidad en el desarrollo del mismo. Todas estas medidas preventivas son hechas con el fin de evitar y descubrir una ruptura precoz y, en tal caso, poder hospitalizar la paciente a tiempo, así como también para que sean observadas directamente e intervenidas tan pronto como sea posible. Del grupo "indicaciones relativas", cuya cesárea. es necesario repetir, se incluyen los siguientes casos:

a) Ruptura de membranas y cabeza flotante.

b) Desproporción relativa.

c) Cesáreas previas con post-operatorio febril.

d) Cicatrices defectuosas.

e) Presentaciones anormales.

f) Separación prematura de la placenta.

g) Placenta previa.

h) Toxemia severa.

i) Nulípara añosa con previas cesáreas.

j) Sufrimiento materno o fetal.

CESAREA DE REPETICION

\begin{tabular}{lllrrrrr}
\hline & & & & 1956 & 1957 & 1958 & Total \\
\hline Electivas . . . . . . . . . . . . . . . . . &. & 14 & 15 & 13 & 42 \\
Frueba de trabajo fallida &. &. &. & 6 & 3 & 11 & 20 \\
Partos obtenidos . . . . . . . . . . . . . & 14 & 25 & 24 & 63 \\
\hline
\end{tabular}

CUADRO NUMERO 7

La prueba de trabajo y sobre todo el encajamiento es uno de los signos favorables "en el parto después de cesárea", razón por la cual en los casos en donde no se consiguió después de un tiempo, se consideraron como pruebas de trabajo fallidas. En el caso de falta de encajamiento nos referimos específicamente a aquelia paciente en la cual no encontramos otra causa que impidiera el descenso de la cabeza. 
Presentamos a continuación las causas que motivaron las cesáreas de repetición, ya sea en forma electiva o las que resultaron por pruebas de trabajo fallidas.

CAUSAS DE CESAREA DE REPETICION

$\begin{array}{lllllllllllllllll}\text { Estrechez pélvica } & \ldots & \ldots & \ldots & \ldots & \ldots & \ldots & \ldots & \ldots & \ldots & \ldots & \ldots & \ldots & 17\end{array}$

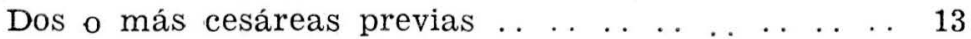

Desproporción céfalo-pélvica $\ldots \begin{array}{llllllll} & \ldots & \ldots & \ldots & \ldots & \ldots & \ldots & 11\end{array}$

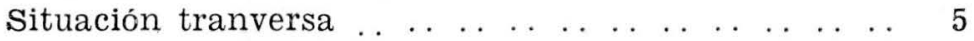

Inminencia de ruptura uterina . . . . . . . . . . . . . . . 4

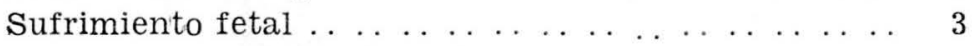

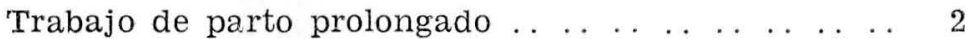

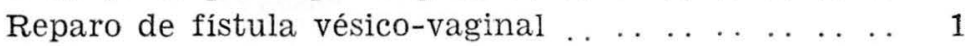

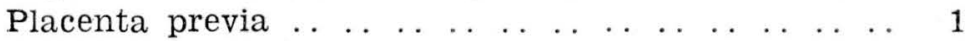

Presentación de pelvis . . . . . . . . . . . . . . . . . . . . 1

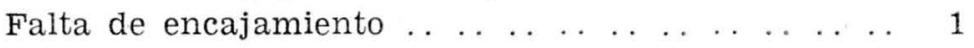

Procúbito de cordón $\ldots \ldots \ldots \ldots$

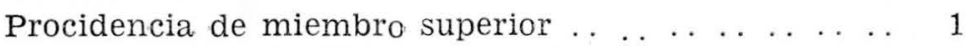

Cirujano e institución desconocidos $\ldots \ldots \ldots \ldots$. . . . . . . 1

CUADRO NUMERO 8

En los casos de cesáreas electivas encontramos una ruptura completa en la únión de la porción segmentaria y corporal.

B) Consideraciones clínicas para el parto después de cesárea

La evaluación clínica para el parto comprende varios aspectos, cuyo juicioso análisis nos dá las normas en el manejo de estas pacientes:

a) Aspecto quirúrgico.

b) Evaluación obstétrica.

c) Evaluación de la cicatriz.

\section{1 - EVALUACION CLINICA}

\section{a). Aspecto quirúrgico}

1. Cirujano e institución.-En los casos en que la cesárea ha sido practicada en un centro altamente especializado, es posible esperar con más seguridad el parto, ya que tanto la indica- 
ción de la cesárea como el procedimiento quirúrgico son más acertados. Es de esperar que un obstetra reconstruya la pared uterina en forma más adecuada que un cirujano o médico general y que el post-operatorio y los controles post-natales sean más cuicadosos.

La institución que pueda poner en práctica los adelantos en anestesia, técnicas quirúrgicas, administración de sangre, antibióticos, control de balance de electrolitos y líquidos, obtendrá mejor cicatrización y por lo tanto disminución en la frecuencia de ruptura (25).

En los casos en que la cesárea ha sido practicada en otra institución es necesario tener una historia completa, la cual debe indicar la paridad, el tipo de pelvis, la indicación, el tipo de cesárea, tamaño del niño, duración del parto, curso del post-parto, etc. La historia obstétrica es de gran ayuda, debido a que la paciente que tuvo uno o más partos después de haber tenido una cesárea es usualmente considerada como candidata para el parto. Por otra parte, aquéllas cuyo primer parto fue por vía abdominal, tendrán menos oportunidad para tener un subsecuente parto. Ambas clases de pacientes deben ser inscritas tempranamente y ciebe obtenerse una historia cuidadosa y detallada.

INSTITUCIONES Y CIRUJANO

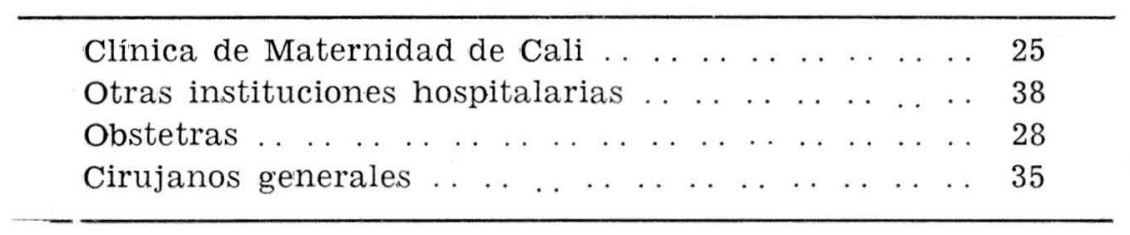

CUADRO NUMERO 9

2. Indicación de la cesárea-Es requisito indispensable que la indicación de la cesárea anterior no haya sido por distocia csea o que la causa no se repita en el presente embarazo. Muchas die estas pacientes han sido conducidas a cesárea por causas ocasionales o "indicaciones relativas", entre las cuales enumeraremos las principales:

1. Placenta previa.

2. Desprendimiento prematuro de la placenta.

3. Prolapso del cordón.

4. Toxemia.

5. Inercia uterina. 
6. Sufrimiento fetal.

7. Distocia de tejidos blandos.

8. Presentación de pelvis.

9. Situación transversa.

10. Complicaciones médicas y quirúrgicas.

11. Tumor pélvico.

12. Distocia cervical.

INDICACIONES DE LA CESAREA ANTERIOR

1. Causa desconocida .................... . . . 22

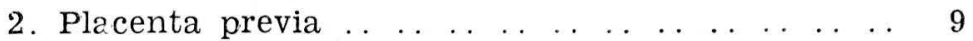

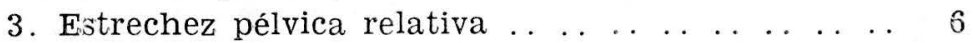

4. Sufrimiento fetal . . . . .

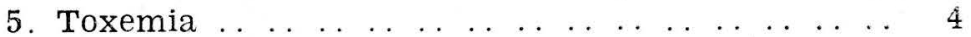

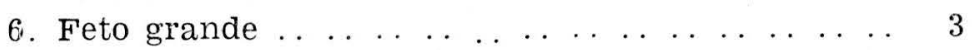

7. Presentación pelvis en nulípara . . . . . . . . . 3

8. Desprendimiento de placenta . . . . . . . . . . . . . 2

9. Trabajo prolongado y distocia de contracción .. 2

10. Desproporción céfalo-pélvica . . . . . . . . . . . . . 2

11. Distocia de cuello . . . . . . . . . . . . . . . . . . . . . . 2

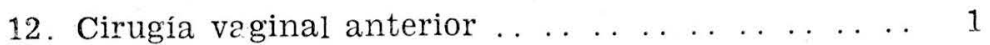

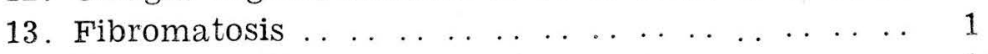

14. Tumor previo $\ldots \ldots \ldots \ldots \ldots$

15. Estrechez pélvica absoluta . . . . . . . . . . . . . . . . 1

$\begin{array}{lllllllllllllllllll} & \text { Total } & \ldots & \ldots & \ldots & \ldots & \ldots & \ldots & \ldots & \ldots & \ldots & \ldots & \ldots & 63\end{array}$

\section{CUADRO NUMERO 10}

NOTA.-Fue posible obtener la historia clinica de algunos de los casos no operados en la Clínica de Maternidad. En los otros la causa de la cesárea se obtuvo del interrogatorio de las pacientes. A aquéllas con historia de estrechea pélvica se les permitió el parto cuando éste fue prematuro.

3.-Tipo de cesárea y procedimiento operatorio.--Es necesario conocer cuál fue la técnica seguida en la práctica de la cesárea para así basar el pronóstico del parto. Existen estadísticas un poco contradictorias, pero la mayoría de ellas están de acuerdo sobre la mayor incidencia de ruptura de la cesárea clásica soore la cesárea segmentaria baja. 
Bruce (7) informa de $38.8 \%$ de partos después de 47 cesáreas sobre un total de 127 casos. De éstas se encontraron 2 rupturas de la cicatriz uterina, las cuales ocurrieron en casos de previas cesáreas clásicas.

Cosgrove (12) (1951), en una serie de 500 cesáreas, presentó 251 niños tenidos por parto de 179 casos seleccionados, un promedio total de $35.8 \%$. Concluye que la incisión segmentaria baja del útero es una eficiente profilaxis contra las más severas rupturas.

Víctor M. Avilés (2) (1951), en un estudio de 651 pacientes con cesáreas previas, consiguió 429 niños con parto espontáneo. En los casos que informa de rupturas uterinas después de cesárea, éstas ocurrieron antes de haber comenzado el trabajo de parto.

Herbert Schmitz $(42,43,44)$, presenta en una serie de 36.293 partos un promedio de 564 cesáreas, 10 rupturas uterinas, de las cuales 6 pacientes ienían historia de cesárea previa y 4 sin ningún antecedente quirúrgico.

Hidman (24), presenta un estudio de 177 partos en el Boston Lying in Hospital, desde 1928 hasta 1946, mostrando que la ruptura de la cicatriz en caso de cesárea clásica ocurre 16 veces más frecuentemente que en los casos de cesárea segmentaria baja.

Lane y Reid (28) (1953) han manifestado sus dudas en este punto. Después de examinar sus resultados en Boston informan la incidencia de un $3.3 \%$ de rupturas en los casos de cesáreas segmentarias bajas, contra $1.3 \%$ después de cesárea clásica. Parece que la incisión vertical en la porción segmentaria baja fue ntilizada en la mayoría de este material y es posible que ésta pueda ser la causa responsable de una mayor incidencia de dehiscencias en la parte segmentaria.

También Dewhurst (15) (1957), en algunos informes británicos sostiene que la mayoría de opiniones muestran una incidencia de $2.2 \%$ en los casos de cicatrices de cesárea clásica y $0.5 \%$ de la cesárea segmentaria.

Aun cuando encontramos algunas divergencias, casi todos los autores concluyen que la cicatriz de la cesárea segmentaria baja es más segura que la de la clásica. Por esta razón las pacientes que han tenido una incisión clásica pueden tener mayor peligro de ruptura al permitir el parto.

4. Horas de trabajo de parto.-En los casos de pacientes nuliparas deberá interrogarse muy cuidadosamente sobre las horas de trabajo de parto que transcurrieron antes de que le fuera 
practicada la cesárea, así como también el grado de dilatación cervical a que llegó. Es lógico pensar que una nulípara a quien se le practicó cesárea antes de haber tenido una dilatación de cuello conveniente, necesitará trabajo de mayor intensidad para poder borrar y dilatar el cuello. En el caso de pacientes multíparas a quienes se les practicó cesárea por cualquiera de las indicaciones anteriormente vistas, requerirá menor trabajo de parto. Hemos visto que las nulíparas en las cuales la cesárea se hizo después de haber logrado una dilatación satisfactoria del cuello, presentaron el parto en menor número de horas que aquélas que no tuvieron mayor dilatación. Por ejemplo: los casos de presentación de pelvis, sufrimiento fetal, etc., en los cuales la cesárea se decidió a última hora después de una conveniente dilatación del cuello.

5. Curso post-operatorio y puerperio-Debemos tener en cuenta la prevención de la sepsis post-operatoria mediante terapia profiláctica. Es posible de esta manera prevenir defectos que pudieran traducirse en mala cicatrización o debilidad del tejido. Es muy importante que las pacientes que han tenido un curso post-operatorio séptico debido a alguna infección endometrial no deben ser incluídas para parto por la posible debilidad del tejido cicatricial.

Sin embargo, Nicholson (36) no ha encontrado prácticamente una gran diferencia entre una convalescencia febril y un curso post-operatorio normal, estableciendo que este último no \&arantiza en el siguiente embarazo contra posibles rupturas uterinas (Munro Kerr, Operative Obst.).

Poidevin y Bockner (39), en estudios de histerografía después de cesárea, no encontraron relación entre el tamaño de la deformidad cicatricial y los diferentes factores clínicos. tales como edad, paridad, indicación para cesárea, técnica y pirexia postoperatoria.

\section{b) Evaluación obstétrica}

Las pacientes con antecédente de cesárea deben ser enviadas unas semanas antes del término a la "Clínica de Distocias". donde su historia debe ser revisada en forma cuidadosa y detallada, incluyendo el tipo de cesárea, las indicaciones, evolución puerperal, etc. El análisis nos excluye aquellas pacientes con alteraciones en el presente embarazo ya sea para madre o niño, tales como la presencia de miomas, anormalidades en la forma 
del útero, sobredistensión, presentaciones anormales, etc., y dejamos como candidatas únicamente aquéllas que llenan todos los requisitos desde el punto de vista quirúrgico, obstétrico y de evaluación de la cicatriz.

En el presente año la evaluación de estos casos por la "Clínica de Distocias" nos ha evitado muchas "pruebas de trabajo fallidas" y por otra parte ha permitido el parto a un mayor número de pacientes.

Detallaremos a continuación algunos puntos en la evaluación obstétrica que aun cuando parezcan por demás, deben considerarse con algún detenimiento.

1. Proporción céfalo-pélvica y evaluación de la pelvis.-ES requisito indispensable la adecuada revisión clínica $y$, si es el caso, radiológica de la pelvis para descartar cualquier desproporción céfalo-pélvica. En los casos límites es algo temerario conducirlas a una prueba de trabajo y sería conveniente incluírlas centro de las cesáreas electivas de repetición. Solamente los casos en donde con certeza existe una buena proporción céfalopélvica serían candidatos.

En los casos de pacientes multíparas con partos anteriores normales a quienes se practicó cesárea por una causa accidental, es lógico suponer una pelvis normal conociendo el tamaño de los niños anteriores y por lo tanto permitir el parto. En los casos de secundigestantes con antecedentes de cesárea se plantea el problema de la apreciación de la pelvis. En nuestro medio donde aún la radiopelvimetría no es un procedimiento de rutina y donce las dificultades técnicas lo obstaculizan, es necesario practicar un examen clínico detenido apreciando cada uno de los detalies de la pelvis. En nuestros casos esta evaluación es un procedimiento que seguimos de rutina de acuerdo con una hoja en donde representamos gráficamente todos los detalles y según ésta, practicamos la clasificación de la pelvis y hacemos el pronóstico. Ésta evaluación clínica ha sido de una gran ayuda y solamente en los casos de pelvis límites hemos encontrado problemas, pero estos casos no entran en el grupo para permitirles el parto.

2. Paridad.-Hemos dividido los casos en tres grupos, a saber:

1 . Secundigestantes con cesárea anterior (nulíparas).

$2^{\circ}$ Multíparas cuyo último embarazo terminó en cesárea.

$3^{\circ}$ Multíparas con uno a más partos después de cesárea.

Nuestra mayor atención la dirigimos hacia el primer grupo, ya que desconocemos la capacidad pélvica de la paciente y la 
mayoria de ellas no han tenido el suficiente tiempo de trabajo de parto para que se les facilite la dilatación del cuello.

Para las pacientes del segundo grupo los cuidados deben ser preferentemente sobre la "evaluación de las condiciones de la cicatriz" y el "aspecto quirúrgico", ya que los partos anteriores, si los niños han sido de tamaño normal, nos indican que la pelvis es de buena capacidad.

Las pacientes del tercer grupo presentan menor riesgo, ya que los partos que han tenido después de la cesárea son una prueba de la resistencia de la cicatriz. Sin embargo, existen estadísticas que muestran rupturas uterinas después de varios partos, por lo cual no deben descuidarse estas pacientes y hay que conducirlas con los mismos cuidados que a las demás.

PARTO EN RELACION CON LA PARIDAD

\begin{tabular}{llllllllllll}
\hline Nulíparas $\ldots \ldots$ & $\ldots$ & $\ldots$ & $\ldots$ & $\ldots$ & $\ldots$ & $\ldots$ & $\ldots$ & $\ldots$ & $\ldots$ & $\ldots$ & 34 \\
Multíparas con cesárea en el embarazo anterior & $\ldots$ & $\ldots$ & 14 \\
Multíparas con partos después de cesárea & $\ldots$ & $\ldots$ & $\ldots$ & 15 \\
\hline
\end{tabular}

CUADRO NUMERO 11

3. Tamaño fetal y edad del embarazo.-Pacientes con fetos pequeños tendrán una mayor oportunidad de un parto con menos riesgos que aquéllas en las que el niño es de tamaño mayor, aún cuando haya proporción céfalo-pélvica. En la selección de cada caso debe considerarse lo más cuidadosamente posible el tamaño fetal y la edad del embarazo.

PESOS FETALES

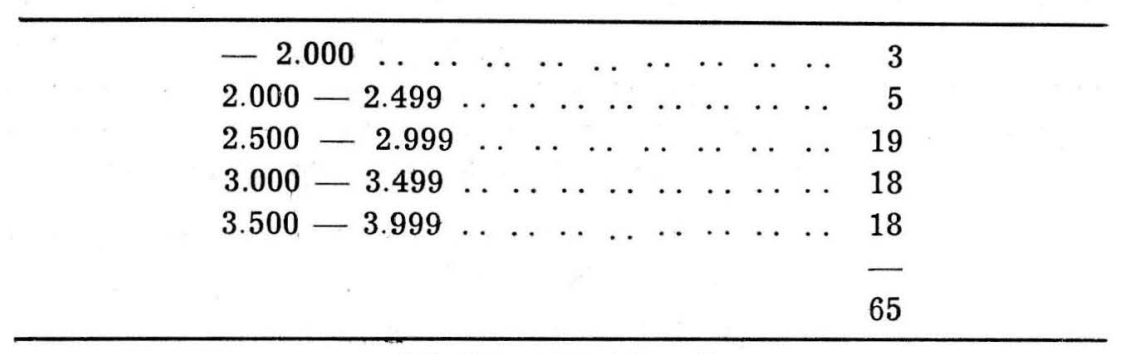

CUADRO NUMERO 12

Nota.-Dos partos fueron gemelares. 
EDAD EN SEMANAS DEL EMBARAZO

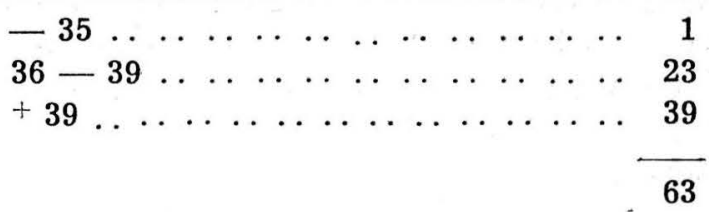

CUADRO NUMERO 13

Una de las causas de mortalidad fetal, cuatro veces mayor en cesárea de repetición que en parto, es el error en la apreciación del tamaño fetal. Resultaron 35\% de niños prematuros en una serie de cesáreas por la falta de apreciación en la edad deI embarazo (45).

La apreciación clínica de los tamaños fetales fue de gran valor, obteniendo partos con fetos menores de 3.500 gramos en más de la mitad de los casos. Es ésta la razón por la cual se pudo obtener partos en varios casos clasificados como "estrechez pélvica límite o relativa" y aún en 1 caso de "estrechez pélvica" (feto de 2.500 gramos).

4. Edad de la paciente.-En la selección de los casos con las secundigestantes nuliparas añosas, debe hacerse la misma consideración que en los casos de mujeres de edad avanzada cuyas estructuras pelvianas presentan una mayor resistencia.

\section{EDAD DE LA PACIENTE}

$\begin{array}{lllllllllllll}\text { De } 15 & \text { a } & 20 & \ldots & \ldots & \ldots & \ldots & \ldots & \ldots & \ldots & \ldots & 14 \\ \text { De } 21 \text { a } & 25 & \ldots & \ldots & \ldots & \ldots & \ldots & \ldots & \ldots & \ldots & 23 \\ \text { De } 26 \text { a } & 30 & \ldots & \ldots & \ldots & \ldots & \ldots & \ldots & \ldots & \ldots & 12 \\ \text { De } 31 \text { a } & 35 & \ldots & \ldots & \ldots & \ldots & \ldots & \ldots & \ldots & \ldots & 7 \\ \text { De } 36 \text { a } & 40 & \ldots & \ldots & \ldots & \ldots & \ldots & \ldots & \ldots & \ldots & 6 \\ \text { De más de } & 40 & \ldots & \ldots & \ldots & \ldots & \ldots & \ldots & \ldots & 1 \\ & & & & & & & & & & \\ \end{array}$

CUADRO NUMERO 14

Los casos de pacientes de más de 35 años son del grupo de las multíparas. 
5. Encajamiento.-Un encajamiento precoz de la presentación y un test positivo de Hillis, hará un caso mucho más favorable que aquéllos en que solamente se produzca después de una prueba de trabajo. Este encajamiento debe considerarse de manera especial en aquellos casos de secundigestantes nulíparas, en donde es posible esperar un encajamiento más precoz que en las multíparas.

En aquellos casos en los cuales no se consiguió el encajamiento después de una "prueba de trabajo" conveniente, se procedió a la "cesárea de repetición". Algunos de estos casos figuran como "pruebas de trabajo fallidas".

6. Variedad de posición.-Los casos cuya variedad es occipito anterior, ya sea izquierda o derecha, los consideramos mejores candidatos, pues las variedades occipito-posteriores requerirán una serie de maniobras que hacen más posible la ruptura uterina, así como el trabajo de parto en la mayoría de los casos será mucho más laborioso y requerirá contracciones de una mayor intensidad. Presentaciones de pelvis o cefálicas anormales no se pueden seleccionar para parto.

PRESENTACION Y VARIEDAD DE POSICION

\begin{tabular}{clr}
\hline & OIA & 41 \\
& ODP & 10 \\
VERTICE 63 & ODA & 6 \\
& OIP & 1 \\
& OIT & 5 \\
& ODT & 0 \\
\hline
\end{tabular}

CUADRO NUMERO 15

Aún cuando nosotros presentamos algunos casos de variedades posteriores, los cuales han tenido una selección cuidadosa y un resultado favorable, no creemos aconsejable que sean consideradas estas pacientes como candidatas óptimas para el parto. En algunos de estos casos en que se permitió evolucionar el parto fue debido al tamaño pequeño del niño.

7. Cuello.-Un "cuello maduro" o sea central, blando, con un buen borramiento y dilatación, nos indica que la paciente ha tenido un "pre-parto" (Alvarez y Caldeyro), y por lo tanto, está en 
condiciones más favorables que aquellas pacientes con cuellos largos y poco borrados. Además las contracciones del pre-parto aún cuando no alcanzan la intensidad de las del parto, sí son. una prueba para valorar por lo menos las condiciones de la cieatriz. La elasticidad del cuello debe notarse ya que en los casos de distocias cicatriciales o anatómicas no estaria muy indicada la prueba de trabajo.

CONDICIONES DEL CUELLO AL INGRESO

\begin{tabular}{rrrr}
\hline Dilatación & \multicolumn{2}{c}{ Borramiento } \\
\hline $3-3$ centímetros & 27 & $-50 \%$ & 7 \\
$3-6$ centímetros & 28 & $+50 \%$ & 56 \\
+6 centímetros & 8 & & - \\
& - & & 63 \\
\hline
\end{tabular}

CUADRO NUMERO 16

El hecho de que un gran número de pacientes a su admisión a la clínica presentaran una dilatación y borramiento del cuello bastante favorable nos explica el por qué fue permitido el parto en los casos en que desconocíamos el cirujano e institución donde se le había practicado la cesárea anterior, así como la causa de ésta.

8. Rayos $X$.-Debe practicársele un estudio de rayos $\mathrm{X}$ no solamente para evaluar la capacidad pélvica y el tamaño fetal, sino practicar una placentografía con el fin de descartar la placenta previa, que sería una contra-indicación formal para permitir el parto.

c) Evaluación de la cicatriz

La incisión uterina no cicatriza por regeneración muscular como sugirió Williams (49) (1917); la cicatriz se produce por formación de tejido fibroso, tal como la descrita por Pheneuf en 1933 y más tarde por Schwartz (46), Paddock (46) y Bortnick (46), en 1938. Con la revisión microscópica del material obtenido por biopsias de la cicatriz (50), en los casos de cesárea de repetición, ha sido posible tener una idea del tipo de regeneración del miometrio. Según Williams y de acuerdo con sus teorías, en algunos casos se prođuce una completa regeneración del miome- 
trio. La cicatriz puede mostrar adelgazamiento o engrosamiento de la unión fibrosa, lo cual está más de acuerda con las ideas expuestas por Schwartz (46). En 20 casos de las biopsias mencionadas en el artículo de Arthur L. Wilson (50) (1951), el autor presenta solamente estos dos tipos de regeneración y dice que la cicatrización parece estar relacionada íntimamente con el cuidado post-operatorio.

Aun cuando Williams Whitridge sostenia que la cicatrización en la cesárea se debía a crecimiento de músculo liso, las investigaciones de Cavaliere (9), Camble, McIntyre (32), Schwartz (46), Paddock (46), Bostnick (46) y Siegle indican todas que el tejido fibroso entra ampliamente dentro de la estructura cicatricial. Si la formación de este tejido fibroso es atacada por la más ligera infección de la cavidad uterina, parece razonable que la cicatrización será débil.

La evaluación cuidadosa de la cicatriz nos dará el pronóstico acerca del parto; por ésto consideramos la cicatriz antes del siguiente embarazo, durante el embarazo, en el trabajo de parto y después del parto.

1. Antes del siguiente embarazo.-Como es difícil evaluar clínicamente la integridad de la cicatriz en la mujer no embarazada, algunos autores (10) han sugerido que la histerografía se haga 3 meses después de la cesárea o el parto con el fin de detectar cualquier defecto de cicatrización o un defecto permanente en la forma. Podevin (39), L. O. S. y Bockner V. Y. (39), sobre un estudio de 43 mujeres sometidas a histerografía, aconsejan que en aquellos casos que demuestran una deformidad en la protundidad de la cicatriz mayor a 5 minímetros, es aconsejable practicar cesárea de repetición. Todos los 43 casos en que se había practicado cesárea presentaban algún grado de deformidad, pero son más importantes los defectos en la profundidad de la cicatriz. No encontraron relación entre el tamaño de la deformidad y los factores clínicos, tales como edad, paridad, indicación para la cesárea, horas de trabajo de parto, técnica operatoria, infección post-operatoria o tiempo en que fue practicada ia histerografía.

Debemos tener en cuenta que a veces ocurren rupturas asintomáticas antes de iniciarse el trabajo de parto, por lo cual se aconseja que toda paciente sea observada cercanamente y que cuando llegue a sus 37 o 38 semanas se hospitalice.

En los casos en que por cualquier circunstancia haya que practicar un legrado, ya sea biopsia o evacuador, debe tenerse en 
cuenta al legrar la pared anterior, con el fin de observar su cicatrización y evitar rupturas.

2. Durante el embarazo.--En los casos en que la paciente no ha sido sometida a las anteriores evaluaciones e inicia otro embarazo, la palpación de la cicatriz uterina puede ayudarnos. En los casos en que encontremos la presencia de persistente sensibilidad o dolor sobre la parte segmentaria del útero, es un signo aue nos indica la posibilidad de tejido cicatricial defectuoso e inminencia de ruptura del útero. La presencia de pequeña hemorragia vaginal o hematuria nos puede indicar inminencia de ruptura o insospechadas rupturas.

Es durante este período cuando se someterá la paciente al estudio de placentografía con el fin de localizar la implantación placentaria.

3. Durante el trabajo de parto.-Las pacientes con antecedentes de cesárea son sometidas a una prueba de trabajo, durante la cual se va a valorar las condiciones de la cicatriz y la evolución del caso. Cuando el trabajo de parto ocurre normalmente, estas pacientes deben ser sometidas a controles rigurosos, para evaluar la intensidad y frecuencia de las contracciones, así como aquellas anormalidades que puedan presentarse durante este tiempo.

Todas estas medidas son tomadas para prevenir una ruptura o para garantizar mayor seguridad en el caso de que la cesárea de repetición esté indicada. Debe oírse regularmente el corazón fetal y si algún signo de sufrimiento fetal se presentara, es necesario hacer la revaluación del caso.

En estas pacientes con frecuencia se presentan algunas irregularidades en la contracción al comienzo del parto. Esta puede ser de poca intensidad y con trastornos en la frecuencia y ritmo. Con la sedación apropiada se previene a menudo la aparición de contracciones secundarias, regularizando el trabajo. En ningún caso las pacientes seleccionadas para trabajo de prueba pueden ser inducidas $(17,29)$, dado el peligro de producir una ruptura uterina.

En nuestros casos con sedación, la mayoría de las veces las contracciones se regularizaron, por lo que consideramos que no sea necesaria la aplicación de Pitocín, máxime en nuestro medio cionde aún no estamos haciendo registros gráficos de la contracción y clínicamente a veces es difícil la apreciación correcta. 
Gran número de pacientes presentaron "malas contracciones" lo que nos permitió observar que la cicatriz de la cesárea anterior influye notoriamente sobre el trabajo de parto. Es un fenómeno aún mal estudiado y que merece toda la atención del caso.

CONTRACCIONES (a la iniciación del trabajo)

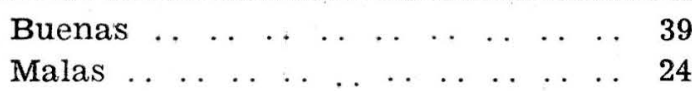

63

CUADRO NUMERO 17

NOTA.-Contracciones buenas $=$ Regulores, ritmicas, buena intensidad y duración.

Contracciones malas $=$ Irregulares, arritmicas, poca intensidad y duración.

La duración del trabajo de prueba depende del encajamiento temprano o del progresivo descenso de la cabeza, de la dilatación obtenida y de la negatividad de los síntomas que signifiquen una posible tendencia a la ruptura. Es lógico suponer que una paciente que no ha tenido parto previo y que está progresando bajo condiciones normales, deba tener un trabajo de prueba más largo que aquélla que ya ha tenido un parto. El promecio de tiempo (16) para un trabajo de prueba en el primer grupo mencionado, no excede de 17 horas, y el promedio de tiempo en pacientes multíparas no debe exceder de 10 horas.

Estas cifras son relativas, pues las diferentes escuelas aún no están de acuerdo sobre el tiempo promedio. Los ingleses son más generosos en la duración del "trabajo de prueba" que las escuelas americanas.

Además, es bastante difícil determinar un promedio de tiempo, debido a que cada paciente en particular es considerada como un caso obstétrico individual. Si el trabajo de parto en tales casos llega a ser irregular y si la dilatación interfiere con éste por una u otra razón, el caso debe ser revaluado con el fin de conocer la posible causa responsable. Si la causa no puede ser corregida de una manera segura y satisfactoria, la paciente debe ser considerada en el grupo de "cesárea de repetición". Si algurios de los síntomas de la ruptura son evidentes, el trabajo de 
parto obviamente ha fallado y la conducta debe ser la cesárea de repetición.

HORAS DE TRABAJO DE PARTO

\begin{tabular}{|c|c|c|c|c|c|c|c|c|}
\hline & & & & $-6 h$. & $6-12 \mathrm{~h}$. & $+12 \mathrm{~h}$ & $+18 \mathrm{~h}$ & $?$ \\
\hline Nulíparas &. & . & . & 4 & 26 & 3 & $(-)$ & 2 \\
\hline Primiparas & . . & . & . & 4 & 7 & 0 & 0 & $(-)$ \\
\hline \multirow[t]{3}{*}{ Multíparas } & . . & . & . & 7 & 9 & 1 & 0 & $\theta$ \\
\hline & & & & - & - & - & - & - \\
\hline & & & & 15 & 42 & 4 & 0 & 2 \\
\hline
\end{tabular}

CUADRO NUMERO 18

Del grupo de "nulíparas", ninguna sobrepasó un trabajo de parto de más de 18 horas y las dos que se encuentran sin dato (?) se debe a que por las contracciones irregulares que tuvieron no fue posible valorar con exactitud la iniciación del trabajo de parto.

De las pacientes multíparas ninguna sobrepasó las 12 horas.

Cosgrove (13), demostró que la sensibilidad y el dolor sobre la cicatriz uterina es un signo de inminencia de ruptura uterina. La hemorragia vaginal durante el primer período del parto, ha sido considerada como un síntoma importante de ruptura o por lo menos de inminencia de ruptura del útero. Otro de los signos al cual se le ha dado bastante importancia es la hematuria, ya que se considera que la vejiga puede estar envuelta en el área cicatricial segmentaria, y por lo tanto sufre los cambios de distensión durante el trabajo de parto. Sin embargo, algunos autores consideran que la hematuria solamente se presenta cuando ha ocurrido la ruptura y por lo tanto no ayuda en la consideración de las condiciones de la cicatriz.

Los latidos fetales y los signos vitales sobre todo el pulso, son de una gran ayuda en la evaluación de la inminencia de ruptura.

La contracción sufre modificaciones grandes, tanto en los casos de ruptura de cesárea clásica como en los casos de segmentaria, y es un signo de gran valor en su apreciación.

4. Después del parto.-En todos los casos de cesárea anterior después del parto debe hacerse una detallada exploración del útero con el fin de evaluar las condiciones de la cicatriz. Se pueden descubrir insospechadas rupturas y sobre todo dehiscencias cie la herida de la cicatriz segmentaria. En 4 de nuestros casos estas dehiscencias tenían un área de 3 a 4 centímetros, cubiertas 
por el peritoneo visceral y no estaban acompañadas de hemorragia. Se les siguió tratamiento a base de antibióticos, ergotrate y reposo. Tres de ellas tuvieron después parto espontáneo nuevamente sin que se hubiera producido ruptura o una mayor dehiscencia del útero, encontrando la cicatriz segmentaria en buenas condiciones.

REVISION DE CAVIDAD

\begin{tabular}{lrrr}
\hline Rupturas & a) Completas & 2 \\
& b) Incompletas (Dehis) & 4 \\
& & Total & 6 \\
\hline
\end{tabular}

CUADRO NUMERO 19

En los casos de dehiscencia incompleta el tratamiento es con servador y solamente hay indicación quirúrgica en los casos en que se presente hemorragia. Los casos de rupturas completas fueron ya descritos cuando se trató lo referente a las rupturas uterinas.

\section{2 - CONDUCCION DEL PARTO}

Las pacientes programadas para parto después de cesárea deben ser hospitalizadas con una semana de anterioridad a la fecha del término del embarazo, ya que solamente bajo condiciones quirúrgicas debe conducirse el parto.

Al ingreso al hospital se practicará clasificación sanguínea y $\mathrm{Rh}$, se harán pruebas cruzadas y se tendrá por lo menos un litro de sangre listo para cualquier eventualidad. Durante el parto, como dedida de precaución, mantenemos una vena canalizada, hacemos la desinfección de piel y, en una palabra, la colocamos en condiciones en que se le pueda practicar una intervención a la mayor brevedad posible. Acostumbramos darle oxigeno a permanencia para mejorar las condiciones fetales.

Se coloca una sonda de Fowley a permanencia, no solamente para control de hematuria sino para mantener evacuada la vejiga y evitarle traumatismos.

Estas pacientes deben ser preparadas psicológicamente con el fin de obtener una buena colaboración durante el trabajo de parto (29), explicándoles las ventajas del parto sobre la cesárea, 
sin descartar la posibilidad de que después de algunas horas de trabajo de parto sea necesario practicar otra cesárea.

La ruptura de membranas debe practicarse tan pronto como las condiciones del caso lo permitan, ya que es de una gran ayuda en la regularización de las contracciones y facilita por otro lado el reconocimiento temprano de hemorragia vaginal o sufrimiento fetal (meconio), signo de gran valor en los casos de ruptura. En pacientes multíparas el encajamiento sucede rápidamente después de la ruptura de membranas.

RUPTURA DE MEMBRANAS

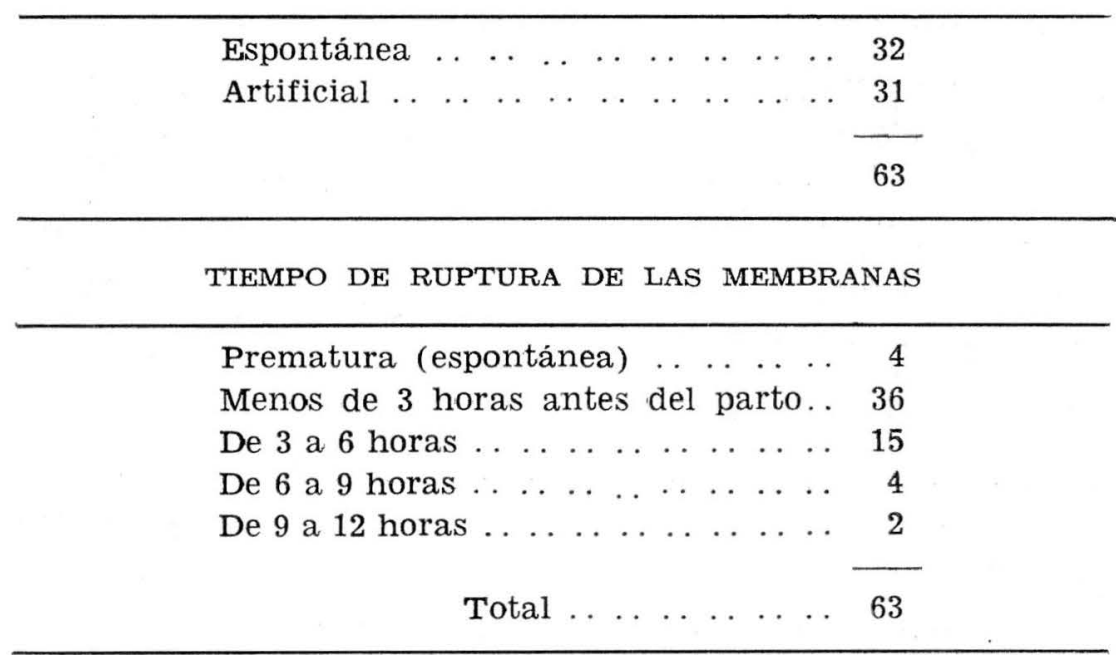

CUADRO NUMERO 20

Hay un gran número de rupturas de las membras unas pocas horas antes del parto, lo cual se debe a que gran parte de estas pacientes entraron en un período avanzado de dilatación.

La conducta en estos casos debe estar encaminada a corregir cualquier tardanza en el periodo expulsivo. Algunos autores creen que el parto debe ser espontáneo después de una ligera sedación. Fleming (19) y Salchi reconocen la necesidad de acortar este período mediante el fórceps bajo y lo practicaron en $7 \%$ y $17 \%$ de los casos, respectivamente.

El grupo presentado por Kenneth y Baker utilizó fórceps en casi el $50 \%$ de los casos. Las ventajas de su utilización son grandes: reducen el período expulsivo por lo menos en 1 hora, disminuyendo la anoxia fetal y se logra un mejor control del periné. 
Además, la anestesia dada para esta intervención puede ser utilizada para la exploración uterina después del parto.

Aun cuando en algunos de nuestros casos se han practicado maniobras de rotación, nosotros creemos que están plenamente desaconsejadas. Tampoco creemos conveniente la estimulación con Pitocín por la mayor incidencia de rupturas uterinas.

CLASE DE PARTO

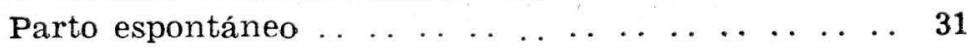
Fórceps:

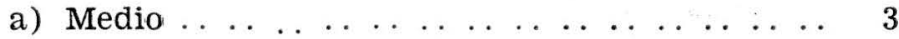

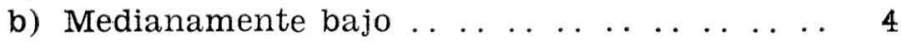

c) Bajo $\ldots \begin{array}{llllllllllllll} & \ldots & \ldots & \ldots & \ldots & \ldots & \ldots & \ldots & \ldots & \ldots & \ldots & \ldots & \ldots & 17\end{array}$

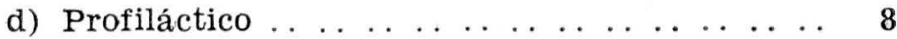

CUADRO NUMERO 21

Entre los fórceps medianamente bajos hubo tres de rotación. Seguimos la clasificación propuesta por Dennen en la aplicación de los fórceps según la estación.

Inmediatamente después del parto en todos los casos debe practicarse la revisión de la cavidad uterina examinando cuidadosamente las condiciones de la cicatriz. Sabemos que muchas de estas rupturas parciales, mejor llamadas dehiscencias, no necesitan tratamiento alguno, aún cuando ciertos tipos de rupturas sí deben ser reparados inmediatamente por la vía abdominal. La evaluación de la cicatriz y la presencia de orificios, depresiones, salientes o adelgazamientos del área cicatricial son signos que van a determinar con mayor exactitud el pronóstico para futuros embarazos.

Las medidas del post-parto son las mismas que ordinariamente se acostumbran: ocitócicos, antibióticos, bolsa de hielo y, además, recomendamos dejar la sonda vesical a permanencia por unas 6 horas. 


\section{SUMARIO}

\section{INTRODUCCION}

\section{I-COMPLICACIONES}

a) Mortalidad materna y fetal.

b) Ruptura uterina.

II-CONDUCTA CON LA PACIENTE DESPUES DE CESAREA
A) Cesárea de repetición.
B) Consideraciones clínicas para el parto des- pués de cesárea.

\section{1 - EVALUACION CLINICA}

A) Aspecto quirúrgico:

1. Cirujano e institución.

2. Indicaciones.

3. Tipo de cesárea.

4. Horas de trabajo de parto.

5. Curso post-operatorio.

B) Evaluación obstétrica:

1. Proporción céfalo-pélvica y evaluación de la pelvis.

2. Paridad.

3. Tamaño fetal y edad del embarazo.

4. Edad de la paciente.

5. Encajamiento.

6. Variedad de posición.

7. Cuello.

8. Rayos X y placentografía.

C) Evaluación de cicatriz:

1. Antes del embarazo:

a) Histerografía.

b) Dilatación y curetaje.

2. Durante el embarazo:
a) Rayos $X$.
b) Placentografía.
c) Clínicamente. 


\section{Trabajo de parto:}

Prueba de trabajo de parto.

Dolor.

Sensibilidad.

Contracciones.

Hematuria.

Hemorragia.

Ruídos fetales.

Signos vitales.

Revisión cavidad.

4. Después del parto

\section{2 - CONDUCCION DEL PARTO.}

\section{MODELO DE HISTORIA CLINICA}

Departamento de Obstetricia - Facultad de Medicina - Universidad del Valle. CLINICA DE MATERNIDAD

Historia $\mathrm{N}^{\circ}$ :

Nombre:

Edad:

Estado civil:

Fecha de ingreso y hora:

Control prenatal:

Causa de ingreso:

EVALUACION CLINICA PARA EL PARTO DESPUES DE CESAREA

\section{A - ASPECTO QUIRURGICO:}

Indicación de la cesárea anterior:

Cirújano: Doctor.

Fecha:

Institución:

Tipo de cesárea:

Horas de trabajo de parto:

Dilatación del cuello alcanzada:

Tamaño fetal:

Curso post-operatorio y puerperio:

B - EVALUACION OBSTETRICA:

Proporción céfalo-pélvica:

Tipo de pelvis:

Paridad:

No del parto después de cesárea:

Tamaño fetal:

General:

Obstetra:

Encajamiento:

Presentación:

gms. Edad del embarazo

Variedad de posición: 
Eorramiento y dilatación del cuello:

Radiopelvimetría:

Placentografía:

C - evaluacion DE LA cicatriz:

Histerografía:

Palpación de la cicatriz:

Dolor espontáneo:

Hemorragia:

Hematuria :

Sufrimiento fetal:

Otros signos:

CONDUCCION DEL PARTO:

Clasificación sanguínea y $\mathrm{Rh}$ :

Sonda a permanencia:

Amniotomía:

Prueba de trabajo: a) corta:

Tipa de parto: espontáneo:

b) media: c) larga:

Horas de trabajo de parto:

Anestesia:

Peso del niño:

fórceps: cesárea de repetición:

Condiciones del niño:

Condiciones de la madre:

Revisión de la cavidad:

Observaciones:

SMD y F. del C.

Analgesia :

gramos.

Clínica de Maternidad.

\section{CONCLUSIONES}

1. El parto en pacientes con cesárea anterior es un procedimiento racional y se puede intentar en aquéllas que reúnan las condiciones necesarias.

2. La evaluación clínica de estas pacientes, considerando los c̀iversos aspectos quirúrgicos, obstétricos y las condiciones de la cicatriz, es un método bastante seguro para permitir el parto.

3. "La Clínica de Distocias" y una instrucción adecuada a los residentes y demás personal, permiten conducir al parto a un mayor número de pacientes con antecedente de cesárea, en una forma más segura.

4. "La prueba de trabajo" llevada con riguroso control, determina la conducta en el parto.

5. La conducción ael parto debe hacerse evitando el esfuerzo del período expulsivo mediante la aplicación de fórceps, y luego debe practicarse la revisión de la cavidad, en todos los casos. 
6. La cesárea de repetición no evita el riesgo de las rupturas uierinas.

7. En las pacientes con antecedente de cesárea, que fueron seleccionadas para parto, no se presentó mortalidad materna.

8. La mortalidad fetal es casi igual entre las pacientes a quienes se practicó cesárea de repetición y a las que se les permitió el parto.

9. En los casos de dehiscencias incompletas debe adoptarse una conducta conservadora.

\section{BIBLIOGRAFIA}

1. AVILES, V. M.-Am. J. of Obst. \& Gynec. (Supp. Vol.) 61A:309, 1951.

2. BAK, T. F., and HAYDEN, G. B.-Am. J. of Obst. \& Gynec. 70:961, 1955.

3. BAUMANN, J.-Ber Gynak. 38:570, 1939.

4. BENAVIDES DE ANDA, L.-Arch. Hosp. Leonardo Martínez (Honduras). 3:15, 1955.

5. BENGTASON, L. P.-Acta Gyn. Obst. Scand. 33:318, 1954.

6. BIRNBAUM, S. J.-Obstet. Gynec. 7:611, 1956.

7. BRUCE, D. F.-Excerta Med. (Sec. X) 7:248, 1954.

8. BURKONS, H. F.-Am. J. of Obst. \& Gynec. 42:75, 1941.

9. CAVALIERE, M.-Ber Gynak. 30:301, 1939.

10. CODY, M. L.-Am. J. of Obst. \& Gynec. 62:415, 1951.

11. CONTI, E. A.-Am. J. of Obst. \& Gynec. 60:851, 1950. 1951.

12. COSGROVE, S. A.-Am. J. of Obst. \& Gynec. (Supp. Vol.). 61A:307,

13. COSGROVE, S. A.-Transactions of the fifth American Congress on Ob. and Gyn. Suplementary volume to the Am. J. of Obst. and Gyn. 64:545, 1952.

14. DELALANDE, A. Bull.-Fed. Soc. Gyn. Obst. 6:323, 1954.

15. DeWHURST, C. J.-Obstet. Gynec. Brit. Emp. 64:113, 1957.

16. DUCKERING, F. A.-Am. J. of Obst. \& Gynec. 51:621, 1946.

17. FLEMING, AMY M.-Am. J. of Obst. \& Gynec. 71:1.902, 1956.

18. FRASER, D.-Proc. R. Soc. Med. 47:119, 1953.

19. FLEMING A. M.-Am. J. of Obst. and Gyn. 71:1.202, 1956.

20. FusCO, T.-Riv. Obst. Gin. Prat. 36:83, 1954.

21. GRASSET, J., and BRET, J.-Excerta Med. (Sec. X). 6:520, 1953.

22. HARRIS, Jr. J. R.-Am. J. of Obst. \& Gynec. 66:1.191, 1953.

23. HERD, S. B.-J. Obst. \& Gynec. Brit. Emp. 60: 237, 1953.

24. HINDMAN, D. H.-Am. J. of Obst. \& Gynec. 55:273, 1948. 
25. JESURUN HAROLD y SIMPSON J.-Am. J. of Obst. and Gynec. $75: 401,1958$.

26. KALAJA, S., and KINNUNEN, O.-Excerta Med. (Sec. S). 7:20, 1954.

27. KISTNER, R. W.-Am. J. of Obst. \& Gynec. 61: 109, 1951.

28. LANE F. R. and REID, D. E.-Obst. Gynec. 2:54, 1953.

29. LAWLER, P., BULFiN, M., LEULER, F.-Am. J. of Obst. and Gyn. 72: $253,1956$.

30. LAWRENCE, R. F.-J. Obst. \& Gynec. Brit. Emp. 60:237, 1953.

31. LOW, D. M.-Am. J. of Obst. \& Gynec. 61:1.957, 1951.

32. McINTYRE, D.-Proc. R. Soc. Med. 17:131, 1924 (Obstet. and Gynaec. Section).

33. McLEAN, L. F.-Am. J. of Obst. \& Gynec. 60:860, 1950.

34. MEREDITH B. S.-Am. J. of Obst. and Gynec. 70:85, 1955.

35. MUÑOZ, S. COBO E.-Cesárea publicación mimeográfica Departamento Obstetricia. Universidad del Valle. Octubre, 1958.

36. MUNRO, KERR, J. M. M.-Operative Obstetrics. London: 1956. Pp. $563,568,878,895$.

37. PALINNA, A.-Excerta Med. (Sec. X). 8.252, 1955.

38. PEDOWITZ, P. and SHUARTZ, R. M.-Am. J. of Obst. \& Gynec. Vol. $74: 5: 107,1957$.

39. POIDEVIN, L. O. S. y BOCKNER V. Y.-J. Obst. and Gynaec. Brit. Emp. LXV: 278, 1958.

40. QUIGLEY, J. K.-Am. J. of Obst. \& Gynec. 17: 597 and 715, 1929.

41. SANTA CRUZ PACHECO F.-Rev. Cubana Obst. Gin. 15:155, 1953.

42. SCHMITZ, H. B.-Am. J. of Obst. \& Gynec. (Supp. Vol.). 61A:289, 1951.

43. SCHMITZ, H. E.-Am. J. of Obst. \& Gynec. 61:1.232, 1951.

44. SCHMITZ, H. E. and BABA, G. R.-Am. J. of Obst. \& Gynec. 57:669, 1949.

45. SCHNEIDER B.-Am. J. of Obst. \& Gynec. 68:1.065, 1954.

46.SCHWARTZ, O. H. PADDOCK, R., and BORTNICK, A. R.-Am. J. of Obst. \& Ginec. 36:962, 1938.

47. SIEGEL, I.-Am. J. of Obst. \& Gynec. 64:301, 1952.

48. TREBICKA, B.-Gin. Polska. 24: 445, 1953.

49. WILLIAMS, J. WHITRIDGE, JOHN HOSPKINS.-Hosp. Bull 28:335, 1917.

50. WILSON, A. L.-Am. J. of Obst. \& Gynec. 62: 1.225, 1951.

51. WINCHESTER, G., and BROWN, R.-Am. J. of Obst. \& Gynec. 70: 923, 1955.

52. WINCHESTER, G., and BROWN, R.-Edinburgh Med. Journal. Vol. 61, Nov. 54:63.

53. WOLF J. R. y ROTER A.-69:1.070, 1955. Am. J. Obst. and Gyn. 